\title{
Prognostic Analysis of Primary Pulmonary Malignant Mesenchymal Tumors Treated Surgically
}

\author{
Muhammet Sayan, M.D., Aykut Kankoc, M.D., Dilvin Ozkan, M.D., Ali Celik, M.D., Ismail Cuneyt Kurul, M.D., \\ Abdullah Irfan Tastepe, M.D.
}

Department of Thoracic Surgery, Faculty of Medicine, Gazi University, Ankara, Turkey

\author{
ARTICLE INFO \\ Received April 19, 2021 \\ Revised May 31, 2021 \\ Accepted June 15, 2021 \\ Corresponding author \\ Muhammet Sayan \\ Tel $90-5071927600$ \\ Fax 90-3122025890 \\ E-mail muhammets@gazi.edu.tr \\ ORCID \\ https://orcid.org/0000-0002-5402-9031
}

\begin{abstract}
Background: Primary pulmonary malignant mesenchymal tumors are rare, constituting only $0.4 \%$ of all lung cancers. Since sarcomas are chemo/radio-resistant, surgical resection is the optimal treatment choice for patients with suitable medical conditions and tumor stage. In the present study, we analyzed the surgical outcomes and survival of primary pulmonary malignant mesenchymal tumors treated surgically.

Methods: We retrospectively examined the records of patients with primary pulmonary malignant mesenchymal tumors who underwent surgical resection at our department between January 2010 and December 2020. Patient data were analyzed according to age, sex, tumor grade and stage, resection completeness, surgical type, and tumor histopathology.

Results: Twenty patients were included in the study. There were 13 men (65\%) and 7 women (35\%). The median survival rate was 36 months (range, 19-53 months), and the 5-year overall survival rate was $37 \%$. Unfavorable prognostic factors for overall survival included parietal pleural invasion $(p=0.02)$, high tumor grade $(p=0.02)$, advanced tumor stage $(p=0.02)$, and extensive parenchymal resection (pneumonectomy and bilobectomy, $\mathrm{p}=0.01$ ). The median length of disease-free survival was 31 months (interquartile range, 21-41 months), and the 5-year disease-free survival rate was 32\%. The most unfavorable prognostic factors for recurrence were parietal pleural invasion $(p=0.02)$, high tumor grade $(p=0.01)$, and tumors requiring lung resection with chest wall resection $(p=0.02)$.

Conclusion: Primary malignant mesenchymal lung tumors are aggressive and have a high mortality rate. However, acceptable overall and disease-free survival rates can be obtained with surgical therapy.
\end{abstract}

Keywords: Malignant, Mesenchymal, Sarcoma, Pneumonectomy, Lung cancer

\section{Introduction}

Primary pulmonary malignant mesenchymal tumors (PPMMTs) are rare, accounting for $0.4 \%$ of all lung cancers according to the literature. Sarcomas detected in the lungs are often metastatic, and PPMMTs have generally been reported in case reports or small case series [1]. Terminological confusion may exist between PPMMTs and pulmonary sarcomatoid carcinomas, including carcinosarcoma, pleomorphic carcinoma, pulmonary blastoma, giant cell carcinoma, and spindle cell carcinoma. Pulmonary sarcomatoid carcinomas are derived from epithelial tissue and include sarcoma components to varying degrees. They were in- cluded in the group of epithelial tumors in the World Health Organization 2004 and 2015 classifications [2]. PPMMTs contain derived mesenchymal cells of the lung parenchyma and are aggressive [3]. Herein, we present an analysis of the surgical outcomes and survival rates of PPMMTs treated surgically.

\section{Methods}

The study was approved by the Gazi University Ethics Committee (IRB approval no., 2021.529). Written informed consent for publication was not obtained from the patients because this was a retrospective study based on medical 
records.

\section{Patient selection}

After the approval of the local ethics committee, the records of patients with pulmonary mesenchymal tumors who underwent surgery at our department were reviewed retrospectively. Patients with metastatic sarcoma, benign inflammatory myofibroblastic tumors, pulmonary sarcomatoid tumors, and sarcomas arising in the chest wall or mediastinum, as well as those whose follow-up records were not available, were not included in the study. Patients who underwent surgical biopsy only for diagnostic purposes were not included in the study, while those with PPMMTs who underwent surgical resection for treatment purposes were included. Patients were clinically staged in the preoperative period. Those with an appropriate clinical stage and medical condition for surgery and resectable tumors were considered suitable for surgery. Clinical staging was conducted based on a physical examination, thoracic and abdominal computed tomography, cranial magnetic resonance imaging and positron emission tomographycomputed tomography, endobronchial ultrasound-guided transbronchial needle aspiration, and mediastinoscopy if required. Patients with metastatic cancer, those who were medically inoperable, and those with unresectable masses were referred to chemoradiotherapy. Adjuvant treatment was not given to patients who underwent complete resection (R0) and had low-grade tumors. Adjuvant chemoradiotherapy was planned for those with microscopic incomplete resection or lymph node metastasis. Other patients received adjuvant chemotherapy. Patient data were analyzed according to age, sex, type of surgery, tumor diameter and stage, resection completeness, and whether adjuvant therapy was applied.

\section{Statistical analysis}

All analyses were conducted using IBM SPSS ver. 20.0 (IBM Corp., Armonk, NY, USA). Descriptive data were expressed as median (range), mean \pm standard deviation (SD), or number and percentage. The chi-square test was used for categorical variables, and the log-rank test was used for continuous variables. The distributions of numerical data were evaluated with histograms and the KolmogorovSmirnov test. Mean values with SDs were used for variables with a normal distribution and median values with ranges (minimum-maximum) were used for those with a skewed distribution. The overall survival (OS) time (months) was calculated from the surgery date to the date of death for patients who died and the date of the study for those who survived.

Disease-free survival (DFS) was calculated from surgery to the date of recurrence or the study date. The OS and DFS were analyzed using the Kaplan-Meier method. The differences in survival rates between the groups were analyzed using the log-rank test or Cox regression analysis.

Table 1. Characteristics of patients $(n=20)$

\begin{tabular}{|c|c|c|}
\hline Characteristic & Category & Value \\
\hline Age (yr) & & $43(19-65)$ \\
\hline Diameter (cm) & & $4.1(1.4-10.0)$ \\
\hline \multirow[t]{2}{*}{ Sex } & Male & $7(35)$ \\
\hline & Female & $13(65)$ \\
\hline \multirow[t]{3}{*}{ Smoking status } & Never & $5(25)$ \\
\hline & Ex-smoker & $9(45)$ \\
\hline & Active smoker & $6(30)$ \\
\hline \multirow[t]{2}{*}{ Side } & Right & $11(55)$ \\
\hline & Left & $9(45)$ \\
\hline \multirow[t]{3}{*}{ Education level } & Primary school & $7(35)$ \\
\hline & High school & $7(35)$ \\
\hline & University & $6(30)$ \\
\hline \multirow[t]{5}{*}{ Type of surgery } & Lobectomy & $9(45)$ \\
\hline & Pneumonectomy & $4(20)$ \\
\hline & Lung resection with CWR & $2(10)$ \\
\hline & Wedge resection & $4(20)$ \\
\hline & Bilobectomy & $1(5)$ \\
\hline \multirow[t]{2}{*}{ Lymph node status } & NO & $17(85)$ \\
\hline & N1 & $3(15)$ \\
\hline \multirow[t]{9}{*}{ Histopathology } & Myofibroblastic sarcoma & $2(10)$ \\
\hline & Spindle cell sarcoma & $1(5)$ \\
\hline & Myxofibrosarcoma & $2(10)$ \\
\hline & Synovial sarcoma & $3(15)$ \\
\hline & Rhabdomyosarcoma & $2(10)$ \\
\hline & Malignant IMT & $3(15)$ \\
\hline & Hemangiopericytoma & $1(5)$ \\
\hline & Fibrosarcoma & $1(5)$ \\
\hline & Liposarcoma & $1(5)$ \\
\hline \multirow[t]{2}{*}{ Complete resection } & RO & $17(85)$ \\
\hline & $\mathrm{R} 1$ & $3(15)$ \\
\hline \multirow[t]{3}{*}{ Adjuvant therapy } & None & $6(30)$ \\
\hline & Chemotherapy & $10(50)$ \\
\hline & Chemo-radiotherapy & $4(20)$ \\
\hline \multirow[t]{3}{*}{ Stage (eighth TNM) } & I & $6(30)$ \\
\hline & II & $4(20)$ \\
\hline & III & $10(50)$ \\
\hline \multirow[t]{3}{*}{ Pleural invasion } & None & $8(40)$ \\
\hline & Visceral pleura & $10(50)$ \\
\hline & Parietal pleura & $2(10)$ \\
\hline
\end{tabular}

Values are presented as median (range) or number (\%).

CWR, chest wall resection; IMT, inflammatory myofibroblastic tumor; TNM, tumor-node-metastasis. 
The significance analysis and hazard ratios of the independent prognostic factors for OS and DFS were conducted using the multivariate Cox regression method. All analyses were conducted with a $95 \%$ confidence interval (CI). Two-sided p-values were calculated, and p-values $<0.05$ were considered to indicate statistical significance.

\section{Results}

Twenty patients who met the inclusion criteria were included in the study. The general characteristics of the patients are shown in Table 1. The median follow-up period was 36 months (range, 3-105 months). There were 13 men (65\%) and 7 women (35\%). The most common histopathological types were malignant inflammatory myofibroblastic tumors and synovial sarcomas (15\% each). The most common surgical procedure was lobectomy with mediastinal lymph node sampling or dissection in 9 patients (45\%). Complete resection (R0) was achieved in 17 patients (85\%). Postoperative complications specific to lung resections were also observed in our series, including prolonged air leakage in 4 patients (20\%), atrial fibrillation in 2 patients (10\%), pneumonia in 1 patient (5\%), and prolonged thoracotomy pain in 1 patient (5\%).

The median OS was 36 months (interquartile range [IQR], 19-53 months), and the 5-year OS was $37 \%$ (Fig. 1). Age, tumor size, and lymph node invasion had no significant effects on OS (Table 2). However, parietal pleural invasion and stage III tumors based on the eighth tumor-node-metastasis (TNM) staging system were poor prognostic factors for $\mathrm{OS}(\mathrm{p}=0.01$ and $\mathrm{p}=0.02$, respectively) (Table 2, Fig. 2). Additionally, the OS was significantly worse in patients who underwent major parenchymal re-

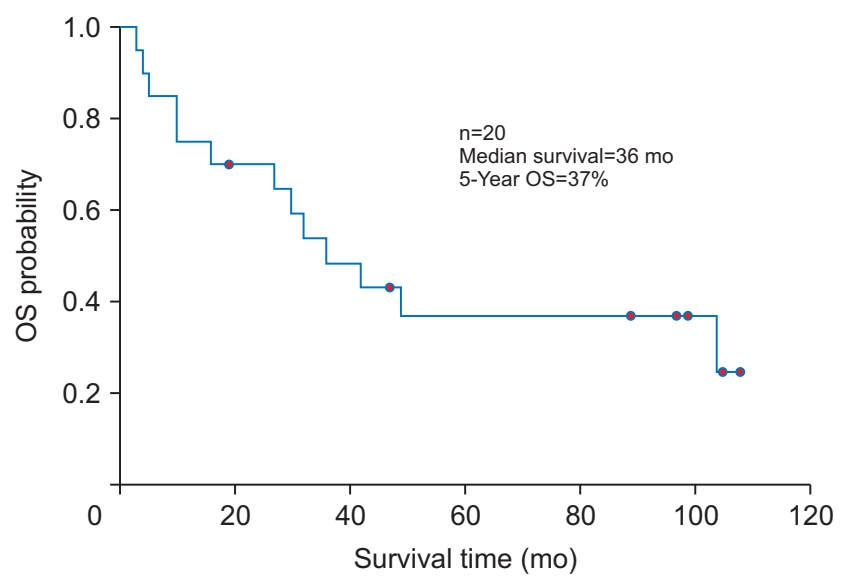

Fig. 1. Survival curve calculated using the Kaplan-Meier method. OS, overall survival. section (pneumonectomy and bilobectomy) ( $\mathrm{p}=0.01)$ (Table 2). Histopathologically, the best median OS was detected in malignant inflammatory myofibroblastic tumors and the worst median OS in the leiomyosarcoma and hemangioperiostoma groups. Nonetheless, the survival difference was not statistically significant (97 months and 10 months, respectively; $\mathrm{p}=0.08$ ). A statistically significant difference was found between high-grade and low-grade tumors defined according to mitosis number, nuclear atypia, and dif-

Table 2. Outcomes of Cox regression analyses for overall survival

\begin{tabular}{|c|c|c|c|}
\hline Variable & $\begin{array}{c}\text { Median } \\
\text { survival (mo) }\end{array}$ & p-value & $\mathrm{HR}(95 \% \mathrm{Cl})$ \\
\hline Sex & & 0.2 & $0.4(0.1-1.4)$ \\
\hline Female & 27 & & \\
\hline Male & 49 & & \\
\hline Diameter (cm) & & 0.01 & $7(1.1-42.0)$ \\
\hline$\leq 5$ & 42 & & \\
\hline$>5$ & 10 & & \\
\hline $\mathrm{N}$ status & & 0.9 & $1.1(0.2-4.6)$ \\
\hline No & 42 & & \\
\hline N1 & 32 & & \\
\hline Pleural invasion & & 0.01 & $4.1(1.1-18.3)$ \\
\hline None & 42 & & \\
\hline Visceral pleural invasion & 32 & & \\
\hline Parietal pleural invasion & 3 & & \\
\hline Age (yr) & & 0.6 & $0.7(0.2-2.2)$ \\
\hline$\geq 55$ & 32 & & \\
\hline$<55$ & 42 & & \\
\hline Stage & & $0.02^{\mathrm{a})}$ & $3.5(1.1-8.9)$ \\
\hline 1 & 49 & & \\
\hline II & 47 & & \\
\hline III & 16 & & \\
\hline Complete resection & & 0.1 & $3.0(0.7-11.8)$ \\
\hline Ro & 47 & & \\
\hline $\mathrm{R} 1$ & 32 & & \\
\hline Adjuvant therapy & & 0.07 & $3.0(0.9-9.9)$ \\
\hline None & 15 & & \\
\hline Chemotherapy & 43 & & \\
\hline Chemo-radiotherapy & 14 & & \\
\hline Surgery & & $0.01^{\mathrm{b})}$ & $3.4(1.1-14.9)$ \\
\hline Lobectomy & 49 & & \\
\hline Pneumonectomy & 4 & & \\
\hline Lung resection with CWR & 27 & & \\
\hline Wedge resection & 36 & & \\
\hline Bilobectomy & 10 & & \\
\hline Grade & & 0.02 & $4.4(1.05-8.3)$ \\
\hline Low grade & 75 & & \\
\hline High grade & 27 & & \\
\hline
\end{tabular}

$\mathrm{HR}$, hazard ratio; $\mathrm{Cl}$, confidence interval; $\mathrm{CWR}$, chest wall resection. a) The patients whose with pathological tumor stage III had poorer

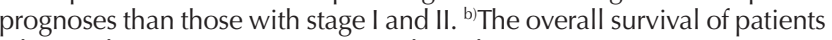
who underwent extensive parenchymal resection (pneumonectomy and bilobectomy) was significantly worse than their counterparts. 


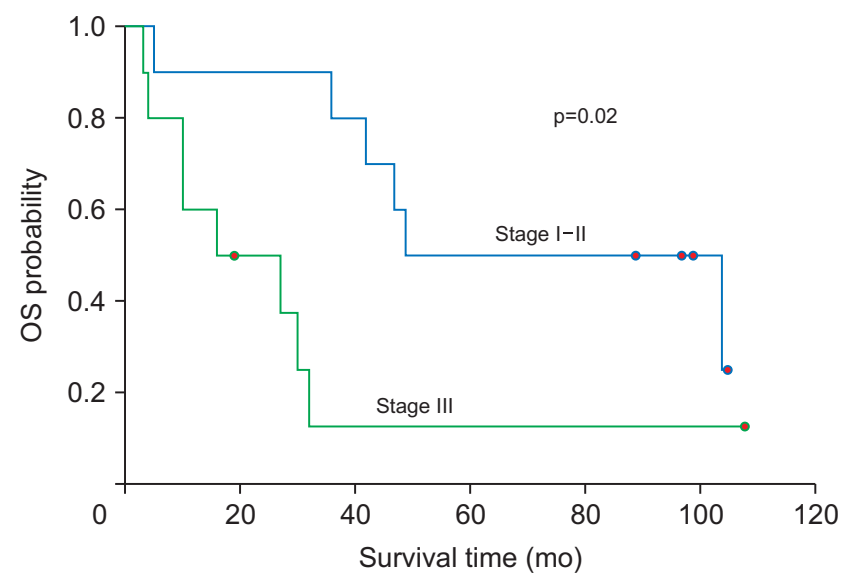

Fig. 2. Comparison of survival rates between patients with early and advanced-stage tumors. OS, overall survival.

ferentiation according to the criteria of the French Federation of Cancer Centers Sarcoma Group-Tumor Differentiation Score by histologic type ( $\mathrm{p}=0.02$ ) (Table 2, Fig. 3). We did not perform low-medium-high or grade $0-3$ subgrouping due to the small number of patients. The criteria for highgrade tumors were a mitosis number of more than 9 in each area, the presence of necrosis, and the presence of differentiation [4].

The median DFS was 31 months (IQR, 21-41 months), and the 5 -year DFS was $32 \%$. The median DFS was significantly worse in patients with parietal pleural invasion $(\mathrm{p}=$ 0.02 ), those who underwent lobectomy with chest wall resection $(\mathrm{p}=0.02)$, and those who had high-grade tumors $(\mathrm{p}=0.01)$. DFS was poorer in female patients $(\mathrm{p}=0.07)$ and those with a high tumor stage $(\mathrm{p}=0.1)$, advanced age $(\mathrm{p}=0.4)$, and fibrosarcoma as the histopathological tumor type ( $\mathrm{p}=0.1)$ than in their respective counterparts; however, those differences were not statistically significant.

\section{Discussion}

In this study, we aimed to present the surgical and survival outcomes of PPMMTs, which are extremely rare. Due to the rarity of PPMMTs, very few studies have investigated their surgical treatment and survival outcomes. In their study, Bacha et al. [1] reported 23 cases with a median age of 51 years and a $65 \%$ proportion of male patients. In a large database study by Spraker et al. [5], the median age was 63 years, and $56 \%$ of patients were men. However, another study reported that male patients were in the minority and had a worse prognosis [6]. In our study, the median age was 43 years, and the percentage of male patients was $65 \%$. Age and sex had no significant effects on OS.

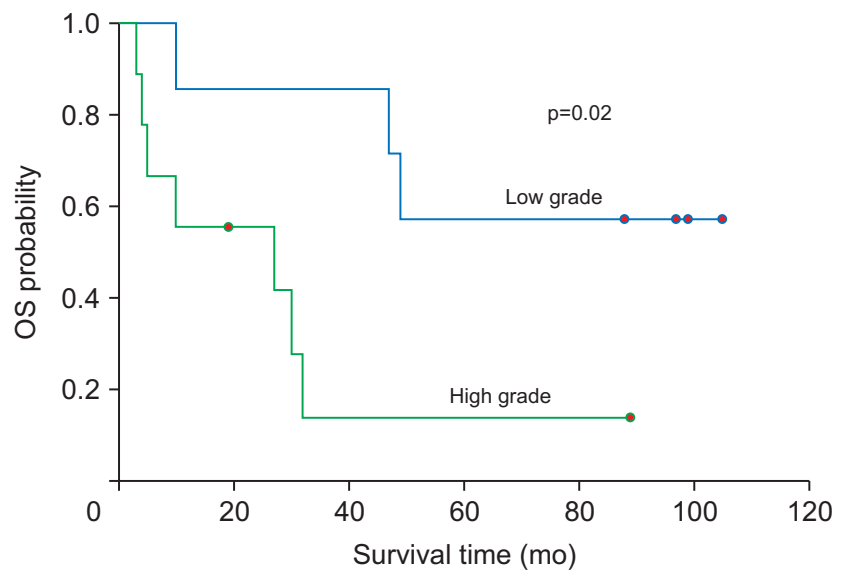

Fig. 3. Graphic representation of the difference in survival rates between patients with low-and-high-grade tumors. OS, overall survival.

As in other sarcomas, R0 resection has been associated with good survival in patients with PPMMTs. The rate of R0 resection was $89 \%$ in a study conducted by Petrov et al. [7]. They reported that $\mathrm{R} 0$ resection was a significant predictor of OS [7]. Similarly, some other studies found that $\mathrm{R} 0$ was a strong marker for OS $[1,6,8,9]$. In our series, R0 resection was achieved in 17 patients (85\%). We found that the median OS was better in the R0 group, but the survival difference was not statistically significant. The reason for this result is the very small number of patients in the R1 resection group.

Generally, poor median and 5-year OS outcomes have been reported in related studies. Robinson et al. [9] reported that the median OS was 39 months, and the 5-year OS was $28.7 \%$. They emphasized that the OS was better in the surgical group [9]. Yamada et al. [8] found that the 5-year OS was 50\%, and significant prognostic factors were tumor diameter, stage, and grade and incomplete resection. The median and 5-year OS were 22 months and 27\%, respectively, in a study conducted by Golota et al. [6], who found that tumor stage and hemoptysis were poor prognostic factors. The results for the median and 5-year OS observed in this study are consistent with the literature.

Typically, regional lymph node metastasis in sarcomas is not expected. Instead, local recurrence or distant hematogenous metastasis is more likely. Spraker et al. [5] reported that the rate of lymph node invasion was $16 \%$, and that lymph node invasion constituted a significant prognostic factor for OS. In other studies, the lymph node invasion rates were between $8 \%$ and $22.5 \%$ [7-9]. Our study showed no metastasis in the $\mathrm{N} 2$ station, and the rate of $\mathrm{N} 1$ invasion was $15 \%$. There was no significant difference in OS be- 
tween the N1 and N0 groups. The negative effect of advanced tumor stage on OS could be explained by tumor diameter rather than lymph node invasion. In addition, our study showed that parietal pleural invasion, an indirect indicator of local invasion, was a negative prognostic indicator for OS and DFS.

Although articles related to non-small cell lung cancer treated surgically have reported pneumonectomy to be a poor prognostic factor, we could not find any studies showing this effect in PPMMTs in the literature $[10,11]$. In our study, the median OS was worse in patients who underwent pneumonectomy. This result may be explained by the larger diameter of the tumors in the pneumonectomy group. However, the better DFS rate of pneumonectomy group contradicts that argument. The literature shows that the DFS of PPMMT patients varies from 15 to 65 months $[3,6-8]$. The DFS of our study is consistent with that reported in the literature, and we found that parietal pleural and chest wall invasion had a negative effect on DFS.

There are several limitations to our study. Principally, this was a retrospective, single-center study that included a small number of cases. In our study, the factors affecting OS and DFS were found as expected. Although no new prognostic factors were found, we think that it is vital to present the surgical and survival results of this rare tumor group.

In conclusion, malignant mesenchymal tumors are aggressive and chemo/radio-resistant. The OS and DFS after surgical resection are acceptable in patients with primary pulmonary sarcoma who have suitable medical conditions and tumor stage.

\section{Conflict of interest}

No potential conflict of interest relevant to this article was reported.

\section{ORCID}

Muhammet Sayan: https://orcid.org/0000-0002-5402-9031

Aykut Kankoc: https://orcid.org/0000-0001-5048-6115

Dilvin Ozkan: https://orcid.org/0000-0002-7149-5982
Ali Celik: https://orcid.org/0000-0001-5385-6492

Ismail Cuneyt Kurul: https://orcid.org/0000-0002-9480-010X Abdullah Irfan Tastepe: https://orcid.org/0000-0002-2032-7444

\section{References}

1. Bacha EA, Wright CD, Grillo HC, et al. Surgical treatment of primary pulmonary sarcomas. Eur J Cardiothorac Surg 1999;15:456-60.

2. Sayan M, Bas A, Valiyev E, et al. Prognostic factors for sarcomatoid carcinomas of lung: a single-centre experience. Lung India 2020;37: 506-10.

3. Duran-Moreno J, Kokkali S, Ramfidis V, et al. Primary sarcoma of the lung: prognostic value of clinicopathological characteristics of 26 cases. Anticancer Res 2020;40:1697-703.

4. Guillou L, Coindre JM, Bonichon F, et al. Comparative study of the National Cancer Institute and French Federation of Cancer Centers Sarcoma Group grading systems in a population of 410 adult patients with soft tissue sarcoma. J Clin Oncol 1997;15:350-62.

5. Spraker MB, Bair E, Bair R, Connell PP, Mahmood U, Koshy M. An analysis of patient characteristics and clinical outcomes in primary pulmonary sarcoma. J Thorac Oncol 2013;8:147-51.

6. Golota J, Osowiecka K, Orlowski T. Primary pulmonary sarcoma: long-term treatment outcomes and prognostic factors. Kardiochir Torakochirurgia Pol 2018;15:162-9.

7. Petrov DB, Vlassov VI, Kalaydjiev GT, et al. Primary pulmonary sarcomas and carcinosarcomas: postoperative results and comparative survival analysis. Eur J Cardiothorac Surg 2003;23:461-6.

8. Yamada Y, Kaplan T, Soltermann A, et al. Surgical outcomes and risk analysis of primary pulmonary sarcoma. Thorac Cardiovasc Surg 2021;69:101-8.

9. Robinson LA, Babacan NA, Tanvetyanon T, Henderson-Jackson E, Bui MM, Druta M. Results of treating primary pulmonary sarcomas and pulmonary carcinosarcomas. J Thorac Cardiovasc Surg 2021; 162:274-84

10. Park JS, Yang HC, Kim HK, et al. Sleeve lobectomy as an alternative procedure to pneumonectomy for non-small cell lung cancer. J Thorac Oncol 2010;5:517-20.

11. Deslauriers J, Gregoire J, Jacques LF, Piraux M, Guojin L, Lacasse Y. Sleeve lobectomy versus pneumonectomy for lung cancer: a comparative analysis of survival and sites or recurrences. Ann Thorac Surg 2004;77:1152-6. 